\title{
Difficulty in differentiating between IgG4-related hepatic inflammatory pseudotumor and intrahepatic cholangiocarcinoma
}

\author{
Ai Hamano ${ }^{1} \cdot$ Reiko Yamada $^{1}$ (i) $\cdot$ Kazunari Kurata $^{1} \cdot$ Junya Tsuboi $^{2} \cdot$ Hiroyuki Inoue $^{1} \cdot$ Kyosuke Tanaka $^{2}$. \\ Noriyuki Horiki ${ }^{2}$ 'Yoshiyuki Takei ${ }^{1}$
}

Received: 5 August 2020 / Accepted: 15 September 2020 / Published online: 9 October 2020

(c) The Author(s) 2020

\begin{abstract}
A 71-year-old man on prednisolone for immunoglobulin (Ig) G4-related renal disease showed increased carbohydrate antigen (CA) 19-9 level; abdominal enhanced computed tomography (CT) showed a lesion in the left lateral segment and dilatation of the peripheral biliary duct. He was referred to our hospital for detailed examination for suspected intrahepatic cholangiocarcinoma. CT and magnetic resonance imaging findings were similar to those for intrahepatic cholangiocarcinoma. However, endoscopic retrograde cholangiopancreatography showed a smooth narrowing of the bile duct which suggested inflammatory disease. Liver biopsy was performed; IgG4-related hepatic inflammatory pseudotumor (IPT) was diagnosed. IgG4-related hepatic IPTs are rare diseases that develop in association with the development of sclerosing cholangitis. Most of these lesions develop in the hepatic hilum and the imaging findings of these tumors are similar to those of hilar cholangiocarcinomas. Thus, hepatic IPTs are difficult to differentiate from malignancy; in some cases, surgical resection has been considered for establishing the diagnosis. In the present case, we could diagnose hepatic IPT on the basis of liver biopsy, which is the recommended approach in cases of suspected hepatic IPT.
\end{abstract}

Keywords IgG4-related disease $\cdot$ Hepatic inflammatory pseudotumor $\cdot$ Intrahepatic cholangiocarcinoma $\cdot$ Liver biopsy

\section{Introduction}

Immunoglobulin (Ig) G4-related disease is a well-known disorder, which can affect various organs of the body, such as the pancreas, biliary tree, liver, kidneys, salivary glands, breast, pericardium, skin, lungs, meninges, and the pituitary gland [1]. IgG4-related disease is characterized by abundant IgG4-positive plasma cell infiltration and high serum IgG4 levels. The disease sometimes manifests as tumorous lesions, and the association between IgG4-related disease and inflammatory pseudotumors (IPTs) has been suggested. The Organizing Committee, comprised of 35 IgG4-related

Reiko Yamada

reiko-t@clin.medic.mie-u.ac.jp

1 Department of Gastroenterology and Hepatology, Mie University Graduate School of Medicine, 2-174 Edobashi, Tsu, Mie 514-8507, Japan

2 Department of Endoscopy, Mie University Graduate School of Medicine, Tsu, Japan disease experts, recommended that "IgG4-related hepatopathy" was a preferred nomenclature instead of hepatic IPT [2].

IgG4-related hepatic IPTs develop in association with development of sclerosing cholangitis [3, 4]. IgG4-related hepatic IPTs are rare disease entities, and it is difficult to distinguish between these IPTs and malignant tumors, such as hilar cholangiocarcinoma or intrahepatic cholangiocarcinoma (periductal infiltrating type). Here, we describe a case of IgG4-related hepatic IPT, diagnosed on the basis of liver biopsy without surgical resection, in which it was difficult to distinguish between IgG4-related hepatic IPT and periductal infiltrating type intrahepatic cholangiocarcinoma.

\section{Case report}

A 71-year-old man was referred to our hospital for detailed examination of a lesion in the liver. He had a history of smoking for 51 years (20 cigarettes per day), no history of alcohol consumption, and no remarkable family history. $\mathrm{He}$ had received treatment for IgG4-related renal disease 
and lymphocytic hypophysitis. He had been taking $4 \mathrm{mg}$ prednisolone daily and $60 \mu \mathrm{g}$ desmopressin acetate hydrate every second day. IgG4-related renal disease had been well controlled, and IgG4 level had been around $180 \mathrm{mg} / \mathrm{dL}$ (5-117 mg/dL). Although he was asymptomatic, his carbohydrate antigen (CA) 19-9 level increased (from $32.4 \mathrm{U} / \mathrm{ml}$ to $304.9 \mathrm{U} / \mathrm{ml}$ ), and abdominal enhanced computed tomography $(\mathrm{CT})$ showed a lesion in the left lateral segment and dilatation of the peripheral biliary duct.

The laboratory data showed elevated levels of hepatobiliary enzymes [aspartate transaminase (AST) 40 IU/1 (normal range; 13-40 IU/l), alkaline phosphatase (ALP) 612 IU/1 (106-322 IU/l), $\gamma$-glutamyl transpeptidase (GTP) $92 \mathrm{IU} / \mathrm{l}$ (13-64 IU/l)] and tumor markers [carcinoembryonic antigen (CEA) $8.4 \mathrm{ng} / \mathrm{ml}(-5.2 \mathrm{ng} / \mathrm{ml})$, CA19-9 $304.9 \mathrm{U} /$ $\mathrm{ml}(-36.8 \mathrm{U} / \mathrm{ml})]$. IgG4 was also elevated; $205 \mathrm{mg} / \mathrm{dL}$. The other parameters were within the normal ranges.

Abdominal enhanced CT revealed ambiguous lesion in segment 3 (S3) of the liver, and dilatation of the
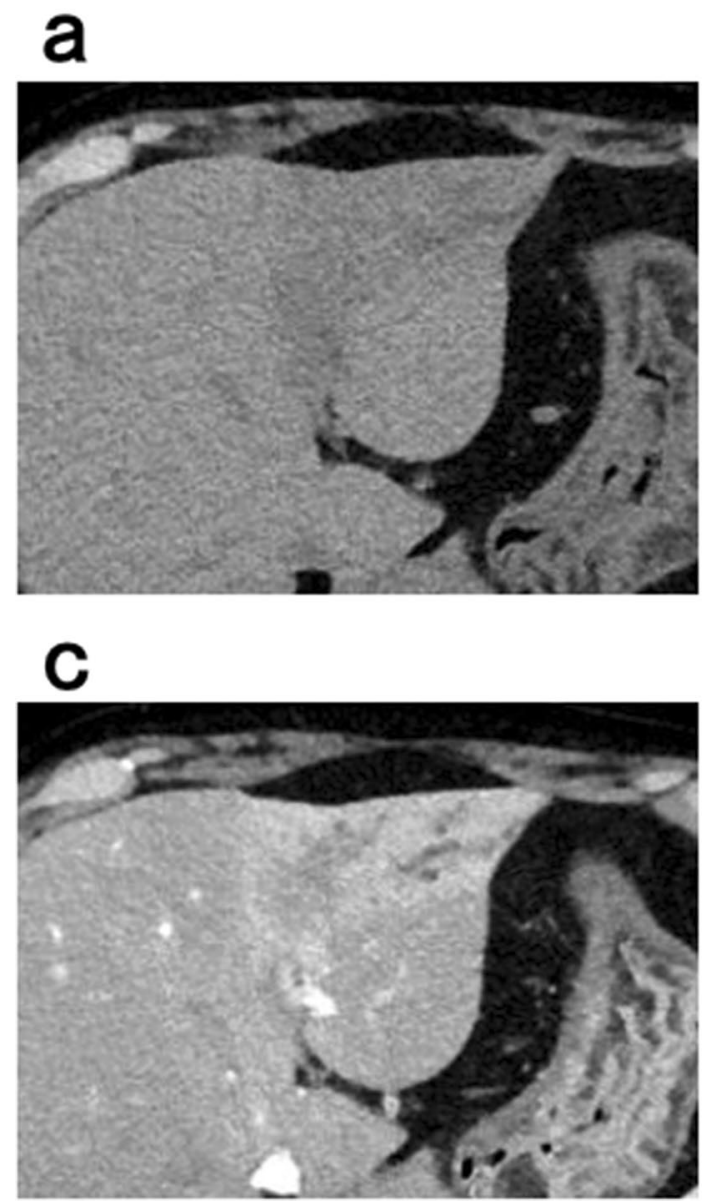

Fig. 1 Abdominal enhanced computed tomography image showing a demonstrated an ambiguous lesion in S3 of the liver and dilatation of the intrahepatic and peripheral bile ducts. The lesion was intrahepatic and peripheral bile duct. The lesion was poorly enhanced on early and portal phase, and showed delayed enhancement on late phase (Fig. 1a-d). Abdominal magnetic resonance imaging (MRI) revealed a lowintensity lesion on a T1-weighted image in $\mathrm{S} 3$ of the liver (Fig. 2a). This lesion appeared as a faint high-intensity poorly marginated mass on a T2-weighted image (Fig. 2b) and a high-intensity mass on diffusion-weighted images with low apparent diffusion coefficient (ADC) values (Fig. 2c). Magnetic resonance cholangiopancreatography (MRCP) showed dilatation of the peripheral bile duct (Fig. 2d). Endoscopic ultrasound (EUS) showed a poorly circumscribed hypoechoic area in S3 and dilatation of bile duct 3 (B3) (Fig. 3a).

Endoscopic retrograde cholangiopancreatography (ERCP) showed smooth narrowing of the bile duct in B3, and a partially beaded appearance of B3 (Fig. 3b). Bile duct biopsy (Fig. 4a) and brush cytology of the bile ducts did not show atypical cells.
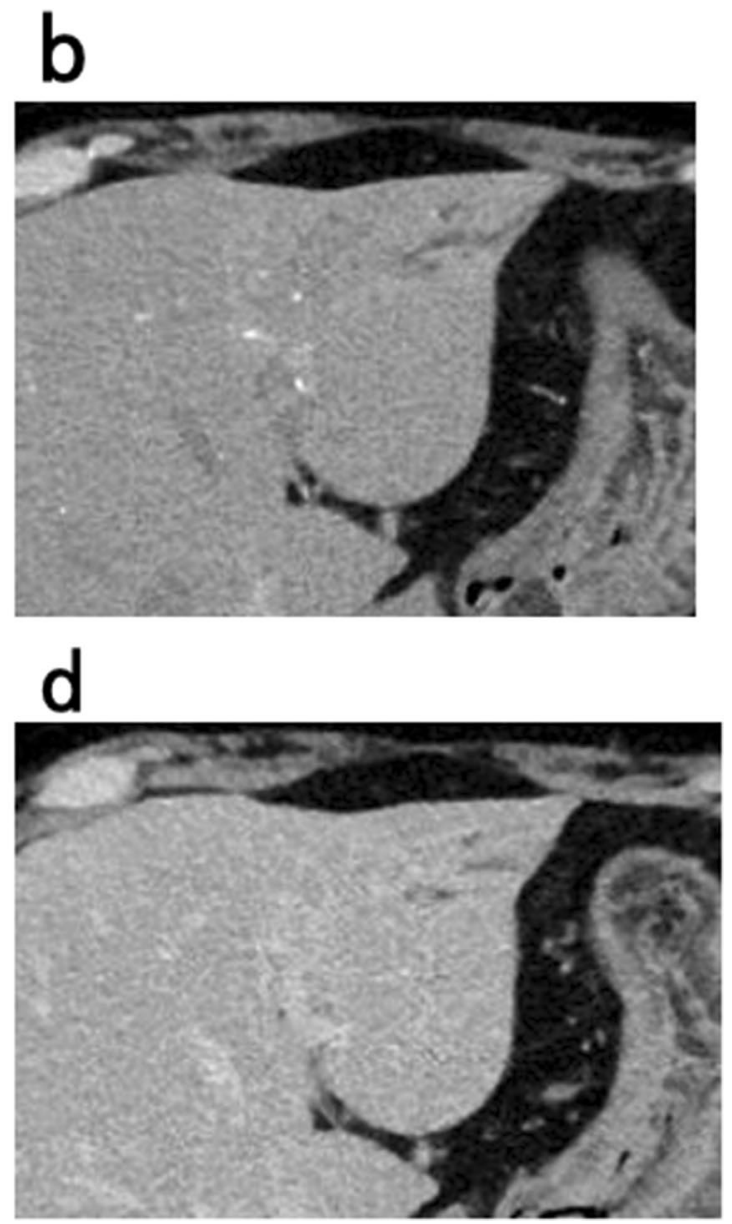

poorly enhanced on $\mathbf{b}$ early and $\mathbf{c}$ portal phase, and $\mathbf{d}$ showed delayed enhancement on late phase 


\section{a}

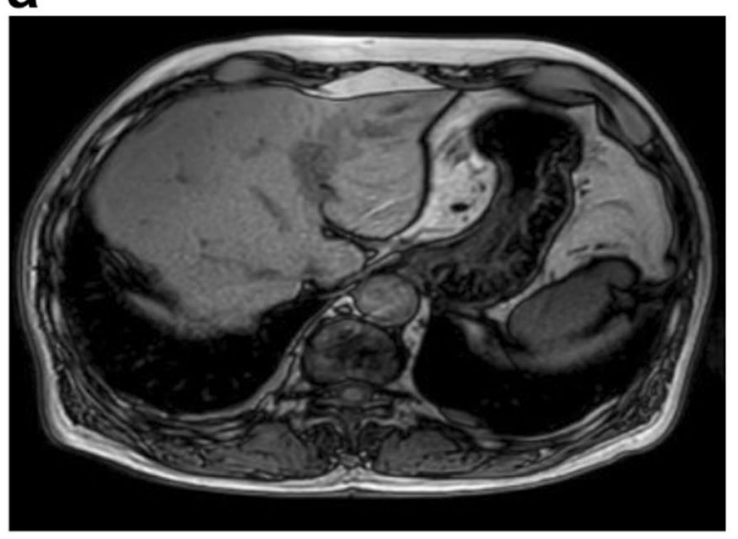

C

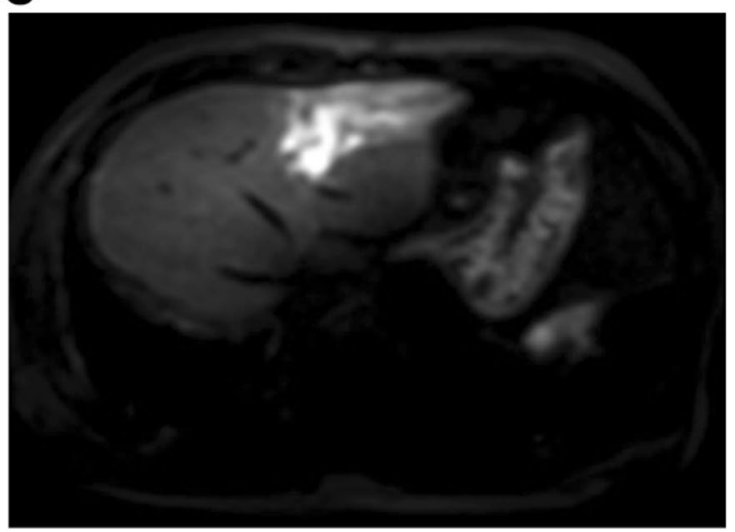

b

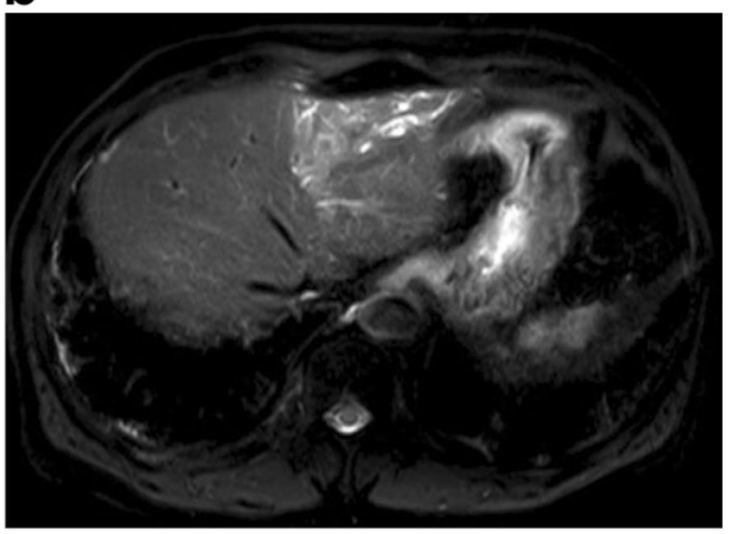

d

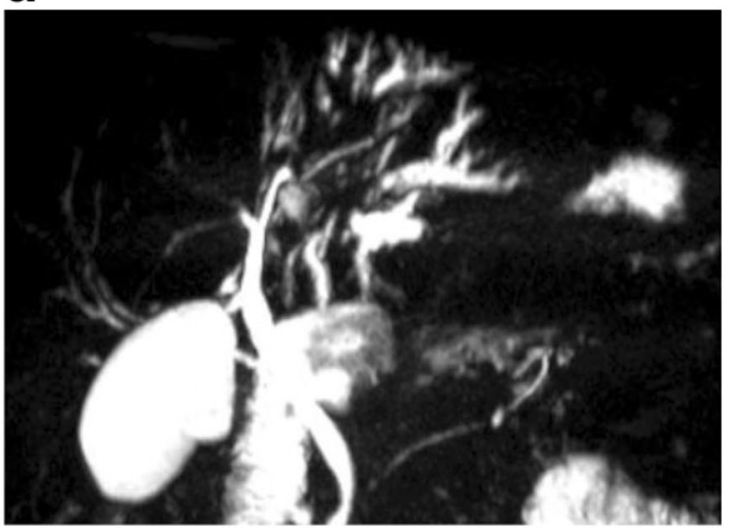

diffusion coefficient value on DWI (c). Magnetic resonance cholangiopancreatography (MRCP) image showing dilatation of the peripheral bile duct
Fig. 2 Magnetic resonance imaging scans showing a low-intensity mass on a T1-weighted image, $\mathbf{b}$ a faint high-intensity mass on a T2-weighted image, a and a high-intensity mass with a low apparent
Fig. 3 a Endoscopic ultrasound (EUS) image showing dilatation of B3 and a poorly marginated hypoechoic area. b Endoscopic retrograde cholangiopancreatography (ERCP) image showing smooth narrowing of the bile duct in B3 and a partially beaded appearance of B3 a

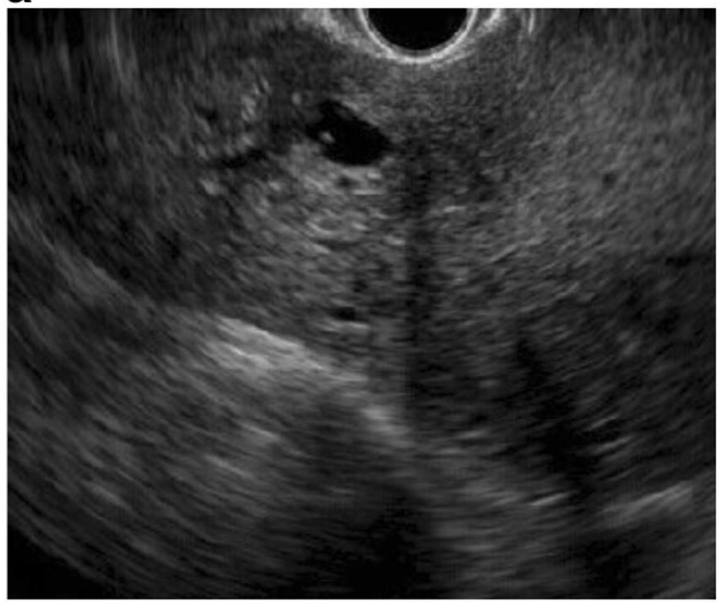

b

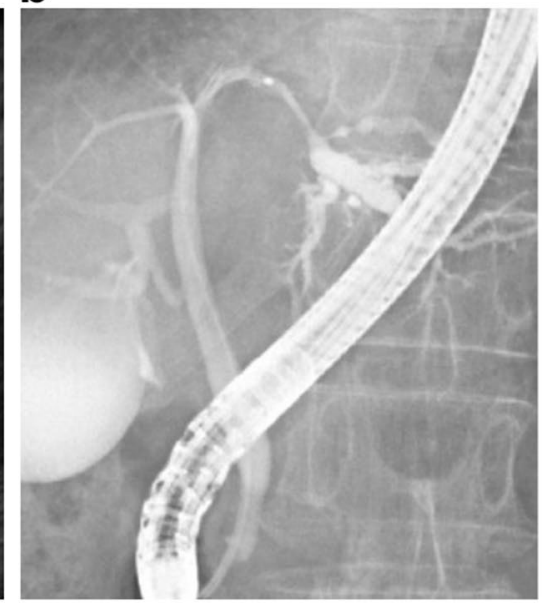

At first, we suspected intrahepatic cholangiocarcinoma (periductal infiltrating type) based on the CT and MRI findings. However, inflammatory disease, like
IgG4-related hepatic IPT, was also suspected because ERCP showed smooth narrowing of the bile duct, bile duct biopsy/cytology did not show cellular atypia, and 

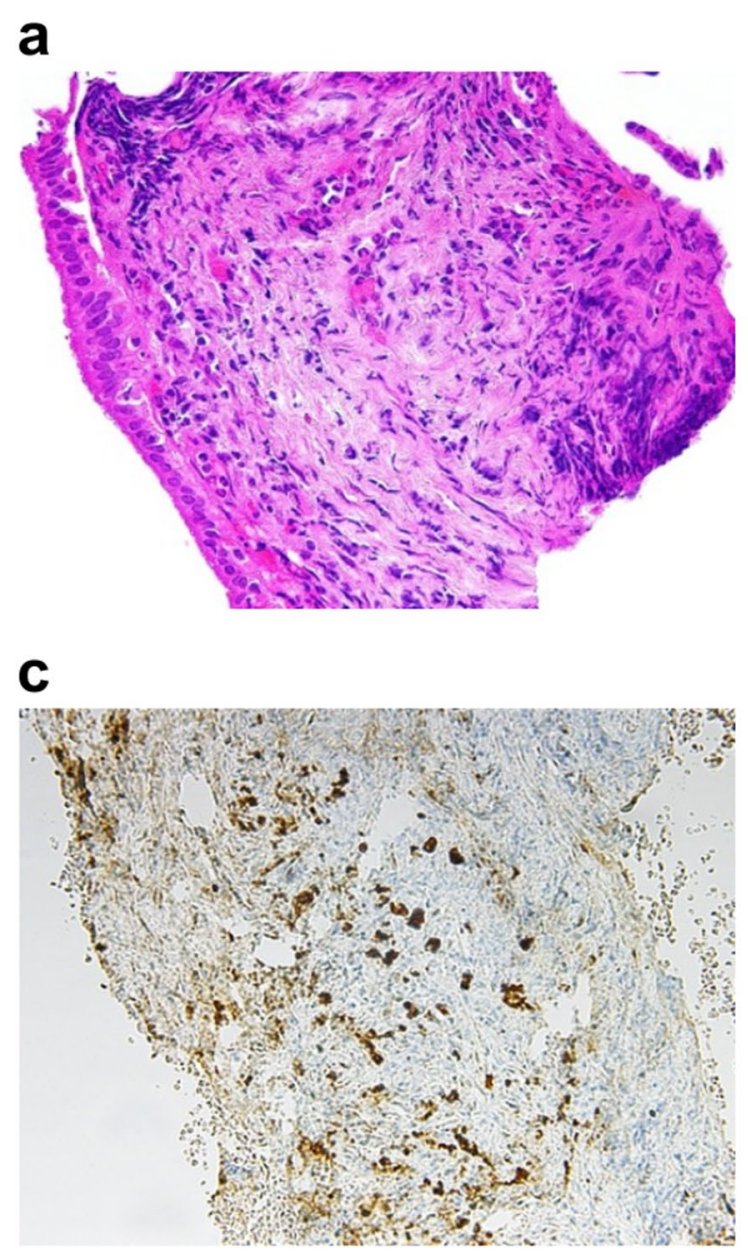

Fig. 4 a Bile duct biopsy image showing absence of atypical cells. b-d: Pathological analysis of hepatic inflammatory pseudotumors. b Inflammatory pseudotumors show inflammatory cell infiltration and

the patient's medical history included IgG4-related disease. Because the biopsy specimen was very small for IgG4 staining, percutaneous liver biopsy was performed for the lesion in S3 using 19G needle (Fig. 5a, b). The pathological findings of liver biopsy were fibrosis with inflammatory cell infiltration (Fig. 4b), IgG4-positive cell infiltration (20 per hpf; Fig. 4c), and obliterative phlebitis (Fig. 4d). Hence, IgG4-related hepatic IPT was diagnosed.

After diagnosis, the patient was administered $10 \mathrm{mg}$ prednisolone daily i.e., the dosage was increased from 4 to $10 \mathrm{mg}$. Two months later, the serum IgG4 level had improved from $205 \mathrm{mg} / \mathrm{dL}$ to $137 \mathrm{mg} / \mathrm{dL}$. The laboratory data of liver function slightly improved. MRI showed a decrease in the size of the mass in S3 and an improvement in inflammation (Fig. 6). One year later, the serum IgG4 level had further improved to $73 \mathrm{mg} / \mathrm{dL}$. b

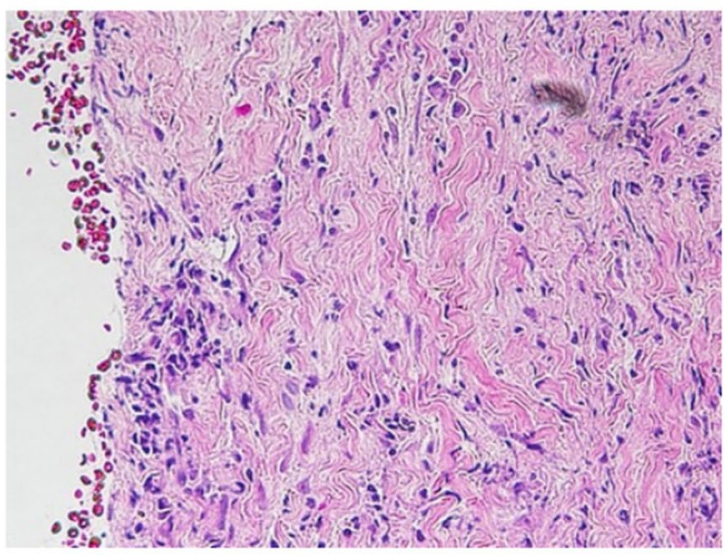

d

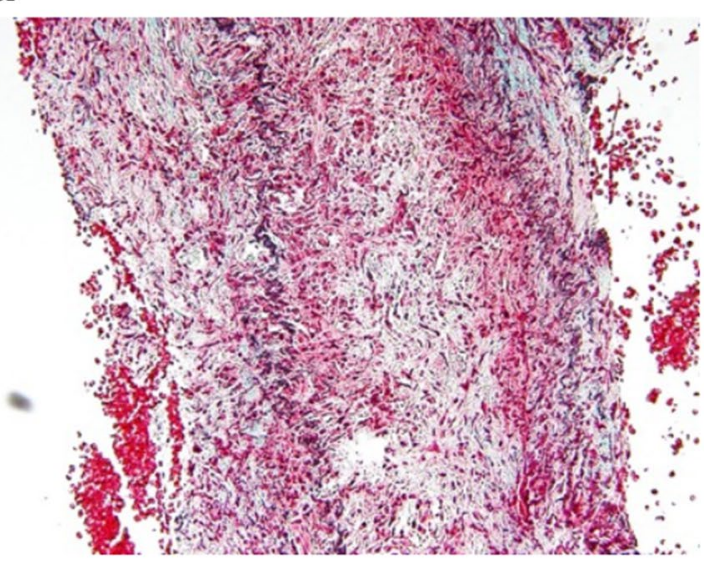

fibrosis. c Inflammatory pseudotumors show IgG4-positive cell infiltration. d Obliterative phlebitis is observed.

\section{Discussion}

Hepatic IPT is pathologically classified into two types, namely, fibrohistiocytic and lymphoplasmacytic IPT [3]. Each characteristic of hepatic IPTs, fibrohistiocytic and lymphoplasmacytic type, is shown in Table 1. Zen et al. characterized fibrohistiocytic IPTs by xanthogranulomatous inflammation, multinucleated giant cells, and neutrophilic infiltration. These tumors mostly developed in the peripheral hepatic parenchyma as mass-forming lesions. The fibrohistiocytic type IPTs manifest as subjective symptoms, such as fever, abdominal pain, and general malaise. In contrast, lymphoplasmacytic IPTs show diffuse lymphoplasmacytic infiltration and prominent eosinophilic infiltration. A significantly greater number of IgG4-positive plasma cells are observed in the lymphoplasmacytic 
Fig. 5 a Echo image showing a poorly marginated hypoechoic area in S3. b Liver biopsy was performed for the lesion in S3 using 19G needle

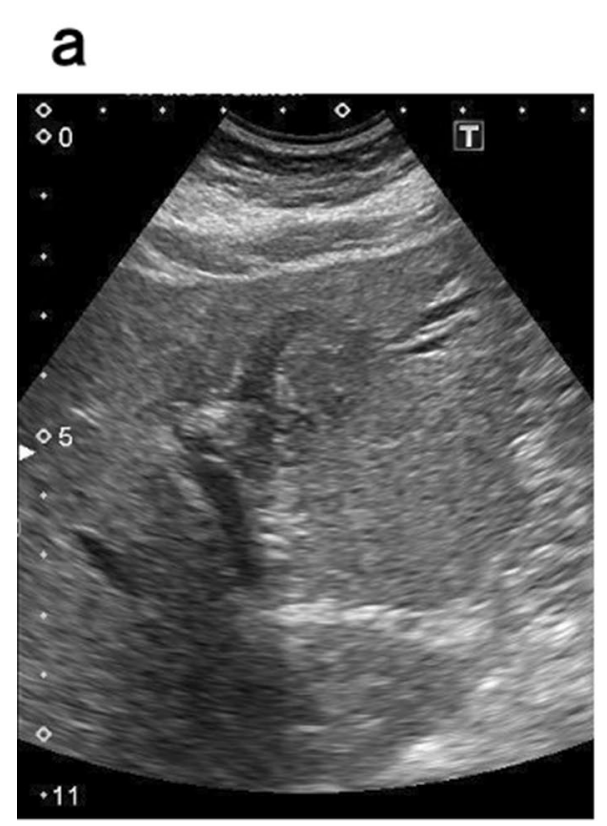

b

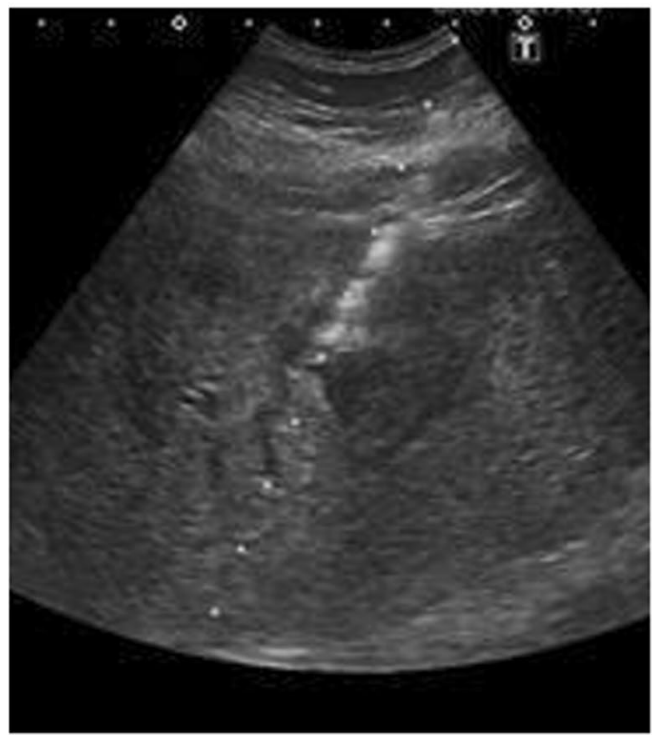

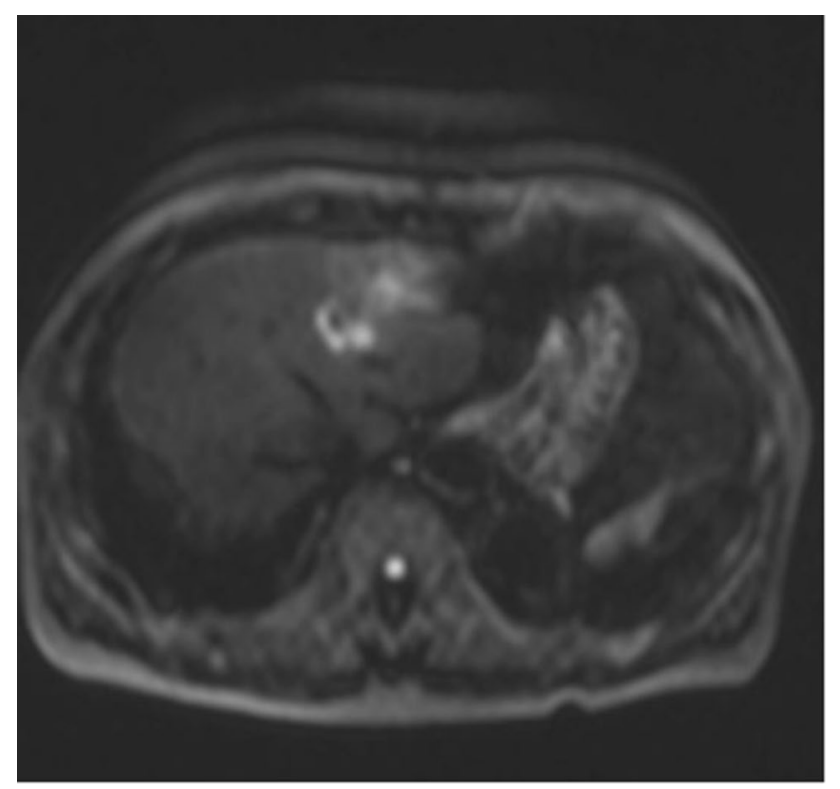

Fig. 6 Two months later, MRI showed the size of the mass in S3 reduced on DWI

IPTs than fibrohistiocytic IPTs. Liver dysfunction was incidentally detected in patients with lymphoplasmacytic IPTs on routine laboratory testing. These tumors usually develop in the hepatic hilum and are distributed along the hilar bile ducts. The lymphoplasmacytic IPTs are similar to hilar cholangiocarcinomas or intrahepatic cholangiocarcinomas (periductal infiltrating type).

IgG4-related hepatic IPTs are rare diseases that develop in association with the development of sclerosing cholangitis
[4]. Most of these lesions develop in the hepatic hilum and the imaging findings of these tumors are similar to those of hilar cholangiocarcinomas. IPT is considered a complication of other diseases, such as autoimmune pancreatitis and sclerosing cholangitis [5]. The pathological characteristics are storiform fibrosis, obliterative phlebitis, eosinophilic infiltration, diffuse infiltration with IgG4-positive cells or plasma cells, and IgG4-/IgG-positive plasma cell ratios $>40 \%[6,7]$. IgG4-related hepatic IPTs are asymptomatic and are sometimes detected concomitantly with other conditions, such as liver dysfunction. Prednisolone is efficacious $[7,8]$ and is associated with better disease prognosis.

It is difficult to differentiate between IgG4-related hepatic IPTs and cholangiocarcinomas, because the imaging findings were similar for the conditions; in some cases, surgical resection was considered for establishing the diagnosis [5]. Histological confirmation on biopsies and accurate diagnosis are important for avoiding the unnecessary administration of treatment, such as surgical resection, to patients with IgG4related hepatic IPTs $[9,10]$. Chougule et al. mentioned that the IgG4-related IPTs diagnosed on biopsies with requisite features showed prompt response to steroids indicating specificity of histopathological findings in predicting treatment response [11]. Thus, to confirm histologically using biopsy sample is important for treatment.

In the present case, we could diagnose hepatic IPT on the basis of liver biopsy, which is the recommended approach in cases of suspected hepatic IPT [12]. Although we initially suspected intrahepatic cholangiocarcinoma (periductal infiltrating type) based on the enhanced CT and MRI findings, ERCP showed smooth narrowing of the bile duct, similar to that noted in inflammatory disease. Furthermore, bile 
Table 1 Clinical characteristics comparison of hepatic inflammatory pseudotumors between fibrohistiocytic and lymphoplasmacytic types

\begin{tabular}{lll}
\hline & Fibrohistiocytic type & Lymphoplasmacytic type \\
\hline Sex & No sex difference & Male > Female \\
Hepatic lobes & Left lobe = Right lobe & Left lobe > Right lobe \\
Location & Peripheral liver & Hilar bile ducts \\
Shapes & Mass-forming type & Periductal infiltrating type \\
Clinical presentation & Subjective symptoms (fever, abdominal & Liver dysfunction by laboratory test \\
& pain, general malaise) & \\
Histological features & Xanthogranulomatous inflammation & Eosinophil infiltration \\
& Multinucleated giant cells & Plasma cell infiltration \\
& Nodular eosinophilic deposition & Russell bodies \\
& Venous occlusion & Obliterative phlebitis \\
& Inflammatory cholangitis & Sclerosing cholangitis \\
\hline
\end{tabular}

duct biopsy and brush cytology findings of the stenotic bile ducts did not show cellular atypia. Hence, we suggest that it is important to include IgG4-related hepatic IPT in the differential diagnosis of liver lesions observed on imaging analysis.

Acknowledgements We are grateful to Dr. Hiroto Yuasa from the Department of Oncologic Pathology, Mie University Graduate School of Medicine, Tsu, Mie, Japan, for helpful discussions.

\section{Compliance with ethical standards}

Conflict of interest Ai Hamano, Reiko Yamada, Kazunari Kurata, Junya Tsuboi, Hiroyuki Inoue, Kyosuke Tanaka, Noriyuki Horiki, and Yoshiyuki Takei declare that they have no conflict of interest.

Human rights All procedures followed have been performed in accordance with the ethical standards laid down in the 1964 Declaration of Helsinki and its later amendments.

Informed consent Informed consent was obtained from the patients for being included in the study.

Open Access This article is licensed under a Creative Commons Attribution 4.0 International License, which permits use, sharing, adaptation, distribution and reproduction in any medium or format, as long as you give appropriate credit to the original author(s) and the source, provide a link to the Creative Commons licence, and indicate if changes were made. The images or other third party material in this article are included in the article's Creative Commons licence, unless indicated otherwise in a credit line to the material. If material is not included in the article's Creative Commons licence and your intended use is not permitted by statutory regulation or exceeds the permitted use, you will need to obtain permission directly from the copyright holder. To view a copy of this licence, visit http://creativecommons.org/licenses/by/4.0/.
2. Stone JH, Khosroshahi A, Deshpande V, et al. Recommendations for the nomenclature of IgG4-related disease and its individual organ system manifestations. Arthritis Rheum. 2012;64:3061-7.

3. Zen Y, Fujii T, Sato Y, et al. Pathological classification of hepatic inflammatory pseudotumor with respect to IgG4-related disease. Mod Pathol. 2007;20:884-94.

4. Koike Y, Aoba T, Maeda M, et al. A case of IgG4-related inflammatory pseudotumor of the liver that had been diagnosed preoperatively as cholangiocarcinoma. J Jpn Surg Assoc. 2017;78:109-13 (in Japanese).

5. Iizuka T, Shibusawa N, Yoshida S, et al. A case of IgG4-associated autoimmune pancreatitis accompanying liver inflammatory pseudotumor. J Jpn Soc Int Med. 2012;101:468-71 (in Japanese).

6. Zen Y. IgG4-related disease of the liver. Kanzo. 2015;56:497-506 (in Japanese).

7. Nakanuma Y, Zen Y. Pathology and immunopathology of immunoglobulin G4-related sclerosing cholangitis: the latest addition to the sclerosing cholangitis family. Hepatol Res. 2007;37:478-86.

8. Ohara H, Nakazawa T, Hayashi K, et al. The concept, diagnosis and treatment of IgG4-related sclerosing cholangitis. JJBA. 2013;27:92-9 (in Japanese).

9. Joshi D, Webster GJ. Biliary and hepatic involvement in IgG4related disease. Aliment Pharmacol Ther. 2014;40:1251-61.

10. Lee JH, Kim HS, Kim JS, et al. Hepatic actinomycosis with immunoglobulin G4-related liver disease. Med (Balt). 2018;97:e11146.

11. Chougule A, Bal A. IgG4-related inflammatory pseudotumor: a systematic review of histopathological features of reported cases. Mod Rheumatol. 2017;27:320-5.

12. Shibata M, Matsubaayashi H, Aramaki T, et al. A case of IgG4related hepatic inflammatory pseudotumor replaced by an abscess after steroid treatment. BMC Gastroenterol. 2016;16:89.

Publisher's Note Springer Nature remains neutral with regard to jurisdictional claims in published maps and institutional affiliations.

\section{References}

1. Kubo K, Yamamoto K. IgG4-related disease. Int J Rheum Dis. 2016;19:747-62. 